\title{
Evaluación de la Resistencia de Unión de Brackets Ortodónticos Fijados a Cerámica de Disilicato de Litio
}

\author{
Evaluation of Union Resistance of Orthodontic \\ Bracelets Fixed to Lithium Dissylate Ceramic
}

\author{
Naiany Macêdo Peixoto'; Jefferson David Melo de Matos²; Valdir Cabral Andrade; \\ Marco Antonio Bottino ${ }^{4} \&$ Lucas Villaça Zogheib 5
}

\begin{abstract}
PEIXOTO, N. M.; DE MATOS, J. D. M.; ANDRADE, V. C.; BOTTINO, M. A. \& ZOGHEIB, L. V. Evaluación de la resistencia de unión de brackets ortodónticos fijados a cerámica de disilicato de litio. Int. J. Odontostomat., 13(2):207-218, 2019.

RESUMEN: El objetivo de este trabajo consistió en evaluar la resistencia al cizallamiento de brackets ortodónticos cementados a una cerámica odontológica a base de disilicato de litio. Fueron confeccionadas 80 muestras rectangulares de cerámica vítrea a base de disilicato de litio. Las muestras fueron divididas inicialmente en 4 grupos de 20 discos según el tratamiento de superficie: ácido fosfórico $37 \%$ por 30 segundos (HP); ácido hidrofluorhidro $10 \%$ por 60 segundos (HF); óxido de aluminio durante 4 segundos (SB); y aspersión con punta diamantada durante 10 segundos (DW). Cada grupo fue dividido en dos subgrupos para la cementación de brackets Edgwise: metálico y cerámico. Los dos nuevos grupos fueron subdivididos en termociclados o no termociclados. Las muestras termocicladas pasaron por 500 ciclos con variaciones de temperatura entre $5{ }^{\circ} \mathrm{C}$ a $55^{\circ} \mathrm{C}$. Los valores numéricos obtenidos en la prueba de cizallamiento se sometieron al análisis estadístico descriptivo (media y desviación estándar), habiéndose realizado la prueba de normalidad Shapiro-Wilk y las pruebas no paramétricas de Kruskal-Wallis y Mann-Whitney. Se verificó que no hubo diferencia estadísticamente significativa en relación al tipo de tratamiento de superficie y la realización o no de termociclado, observándose diferencia estadísticamente significativa sólo en el tipo de bracket, siendo la resistencia de unión en el bracket metálico mayor que en el de cerámica. Así, el tipo de tratamiento de superficie y el envejecimiento por termociclaje no fueron decisivos en la resistencia de unión de brackets ortodónticos a la cerámica de disilicato de litio.
\end{abstract}

PALABRAS CLAVE: cerámica, soportes ortodóncicos, resistencia al corte.

\section{INTRODUCCIÓN}

Al evaluar el contexto actual de salud bucal, con mecanismos más eficaces de prevención y tratamiento de las enfermedades caries y periodontal, es posible percibir un cambio en la demanda y en el perfil de los pacientes que buscan el tratamiento odontológico (Flores et al., 1990; Della Bona et al., 2000). En los últimos años, se ha observado un aumento expresivo en el número de pacientes adultos que presentan restauraciones estéticas y buscan el tratamiento ortodóntico para adecuar la sonrisa (Grewal Bach et al., 2014; Bakhadher et al., 2015).
Ante esta búsqueda por la estética, se observaron mejoras significativas en las propiedades ópticas y mecánicas de los materiales restauradores, especialmente de las cerámicas dentales. Debido a las importantes propiedades, como estética, baja densidad, alta resistencia al desgaste, alta dureza, inercia química, tenacidad a la fractura, excelente resistencia a la corrosión y compresión, baja conductividad térmica, similitud óptica a los tejidos dentales, radiopacidad, estabilidad de color, entre otras, las cerámicas odontológicas han sido consideradas el mejor mate-

\footnotetext{
1 Departamento de Odontología, Facultad Paulo Picanço, Fortaleza - CE, Brasil.

2 Programa de Maestría en Odontología Restauradora, Departamento de Materiales Odontológicos y Prostodoncia, Universidade Estadual Paulista (UNESP), Instituto de Ciencia y Tecnología ICT, Câmpus São José dos Campos, São José dos Campos - SP, Brasil.

${ }^{3}$ Departamento de Odontología, Universidade Federal de Juiz de Fora UFJF, Governador Valadares - MG, Brasil.

${ }^{4}$ Departamento de Materiales Odontológicos y Prostodoncia, Universidade Estadual Paulista (UNESP), Instituto de Ciencia y Tecnología ICT, Câmpus São José dos Campos, São José dos Campos - SP, Brasil.

${ }^{5}$ Departamento de Odontología, Universidade de Fortaleza UNIFOR, Fortaleza - CE, Brasil.
} 
rial para restauraciones indirectas (Caruso et al., 1990; Della Bona et al., 2000; Della Bona \& Di Guida, 2014).

La estructura de las cerámicas está formada por elementos metálicos y sustancias no metálicas, como óxidos, nitruros y silicatos, formando un compuesto muy estable debido a su alta resistencia de unión interatómica primaria (iónica y covalente) (Della Bona \& Di Guida).

El primer relato de utilización de las cerámicas en odontología describió la fabricación de dientes para prótesis total por el dentista Nicholas Dubois y el químico Alexis Duchateau en 1774 (Amoroso et al., 2012). Las cerámicas feldespáticas fueron las primeras en ser confeccionadas en alta fusión, sin embargo, su baja resistencia, responsable de restringir su uso a lugares de pequeña carga oclusal, impulsó a los científicos en la búsqueda de elementos que mejoran su estructura sin causar la pérdida en las características estéticas que hacían el material atractivo.

Las cerámicas feldespáticas fueron las primeras en ser confeccionadas en alta fusión, sin embargo, su baja resistencia, responsable de restringir su uso a lugares de pequeña carga oclusal, impulsó a los científicos en la búsqueda de elementos que mejoran su estructura sin causar la pérdida en las características estéticas que hacían el material atractivo. Con ello, fueron reforzadas por leucita para mejorar sus propiedades, recibiendo así la indicación de uso para facetas laminadas, inlays y onlays. El uso de este material tuvo reflejo en las características mecánicas, pero las cerámicas feldespáticas continuaban presentando resistencia flexural de apenas $180 \mathrm{MPa}$, un valor inferior que aquellos presentados por la incorporación de disilicato de litio o óxido de zirconia (Amoroso et al.). La adición de cristales de disilicato de litio dispersos en una matriz vítrea de forma interlazada en la estructura de las cerámicas feldespáticas favoreció sus propiedades mecánicas. Su resistencia flexural pasó a aproximarse a $400 \mathrm{MPa}$ y no hubo daños estéticos (Instrucciones 68 de uso IPS e.max Press, 2009), lo que posibilitó el uso de esta cerámica hasta los días de hoy.

Los sistemas vítreos reforzados por disilicato de litio poseen en su microestructura un $40 \%$ de litio en forma de cristales de metassilicato insertados en una fase vítrea (Tysowsky et al., 2009). Estos sistemas buscan reproducir la naturalidad de la estructura dental, proporcionando buenas propiedades mecánicas y translucidez, garantizando una restauración indirecta con buena estética y resistencia, características importantes una vez que su indicación engloba tanto dientes anteriores como posteriores (Gehrt et al., 2013).

Entre los materiales cerámicos más indicados actualmente, la cerámica vítrea a base de disilicato de litio ha ganado destaque debido a su versatilidad del sistema que puede ser indicado para región anterior y posterior como restauraciones monolíticas o estratificadas (técnica cut-back), fabricadas vía CAD / CAM o prensado. Su resistencia flexural puede alcanzar $400 \mathrm{MPa}$, y estando asociada a la cementación resinosa permite la confección de restauraciones finas y resistentes. El sistema presenta diferentes variaciones de color y translucidez con óptimas propiedades ópticas (Oliveira et al., 2013).

Considerando el creciente uso de las cerámicas en la cavidad bucal, especialmente la cerámica vítrea a base de disilicato de litio, se puede tener un aumento en la búsqueda de tratamiento ortodóntico de pacientes con restauraciones cerámicas. Muchos pacientes adultos con restauraciones cerámicas cementadas necesitan y / o desean nuevas correcciones de posicionamiento y alineación dental a través de la Ortodoncia, debido al hecho de que el tratamiento ortodóntico previo al tratamiento rehabilitador no fue realizado o porque el movimiento ortodóntico anterior no fue satisfactorio. La ortodoncia indicada correctamente debe ser realizada previamente al tratamiento rehabilitador con facetas, prótesis e implantes, sin embargo, ante la búsqueda acelerada e inmediata por la estética, el correcto orden entre los tratamientos es alterado (Brooks \& Polk, 1998).

La fijación de brackets ortodónticos a superficies cerámicas presenta un porcentaje de falla mayor que la fijación sobre el esmalte. La resistencia de unión de los brackets con la restauración depende del tipo de cerámica, de su condicionamiento superficial, del tipo del bracket (material, diseño de la base y modo de retención), de las propiedades del sistema adhesivo, de la fuente de luz polimerizadora, así como de la habilidad del clínico durante la realización de ese procedimiento (Grewal Bach et al.). Además, en estas situaciones, el desafío no se limita a la unión adecuada del brazalete a la superficie cerámica, pero también su facilidad de remoción para evitar mayores daños a la restauración (Macri et al., 2015).

Buscando mejorar y ampliar las limitaciones de unión, el avance de materiales adhesivos y el desarrollo de nuevas técnicas puede posibilitar la 
PEIXOTO, N. M.; DE MATOS, J. D. M.; ANDRADE, V. C.; BOTTINO, M. A.; ZOGHEIB, L. V. Evaluación de la resistencia de unión de brackets ortodónticos fijados a cerámica de disilicato de lítio. Int. J. Odontostomat., 13(2):207-218, 2019

cementación directa de brackets ortodônticos en otras superficies, sustituyendo el esmalte (Vieira et al., 2002; Masioli et al., 2011; de Matos et al., 2016).

En el presente trabajo se analizaron los resultados obtenidos en el análisis de los resultados obtenidos en el estudio de los resultados obtenidos en el estudio. Por otro lado, se nota una dificultad en la unión química directa de brackets cerámicos compuestos de alúmina (óxido de aluminio) monocristalina o policristalina a los adhesivos utilizados (Della Bona \& Di Guida). Por lo tanto, diversos investigadores han buscado probar diferentes protocolos de tratamiento superficial de cerámicas para favorecer la unión de brackets ortodónticos a las restauraciones cerámicas (Girish et al., 2012; Ebert et al., 2016; Zhang et al., 2016). Varias técnicas se han utilizado para la unión de brackets a las superficies cerámicas y éstas difieren en la preparación de la superficie de la restauración y del agente de unión aplicado. Algunos ejemplos de estas técnicas reportan el uso de ácido fosfórico (Alakus et al., 2016); Y en el caso de las mujeres. En el presente estudio se analizaron los resultados obtenidos en el estudio de los resultados obtenidos en el estudio, y el efecto sobre la rugosidad de la superficie (Zhang et al., 2013; Mehta et al., 2016, Lestrade et al., 2016). En la actualidad, una nueva forma de tratamiento de la superficie cerámica por medio de la utilización de láser ha sido probada (Ahrari et al., 2013; Fornaini et al., 2013; Mirhashemi et al., 2017).

En este sentido, el objetivo del presente estudio fue evaluar la unión de brackets metálicos y cerámicos a la superficie de cerámica vítrea de disilicato de litio después de diferentes tratamientos de superficie.

\section{MATERIAL Y MÉTODO}

La Tabla I presenta los materiales utilizados en la investigación y sus respectivos nombres comerciales y fabricantes.

El presente trabajo fue desarrollado en asociación con el Laboratorio de Materiales Dentales y Prótesis (Odontología Restauradora) del Instituto de Ciencias y Tecnología (ICT-Unesp), São José dos Campos - SP.

Tabla I. Materiales utilizados en la investigación.

\begin{tabular}{|c|c|c|}
\hline Material & Composición & Fabricante \\
\hline Cerámica Disilicato de Litio & $\begin{array}{c}\text { Dióxido de silicio (SiO2); Óxido de litio (Li2O); Óxido de } \\
\text { aluminio (Al2O3); Óxido de Potasio (K2O); Óxido de } \\
\text { Magnesio (MgO); Óxido de Zinc (ZnO); Pentóxido de } \\
\text { Fósforo (P2O5); otros óxidos }\end{array}$ & $\begin{array}{l}\text { Ivoclar Vivadent, Schaan, Liechtenstein } \\
\text { IPS emax CAD/CAM }\end{array}$ \\
\hline
\end{tabular}

Ácido Fosfórico al 37\%

Ácido Fluorídrico al 10\%

Óxido de Aluminio

Puntas Diamantadas

Silano

Bracket metálico

Bracket œrámico

Cemento resinoso

Fotopolimerización
Ácido fosfórico al 37\%, espesante, colorante y agua desionizada

Ácido fluorhídrico al 10\%, agua, espesante, tensoactivo y colorante

Partículas de óxido de aluminio de $50 \mu \mathrm{m}$

$$
\text { Etanol; 3-trimetoxisilil propil metacrilato }
$$$$
-
$$

Cerámica de Alumina, Bi-chorreados Silanizados

Monómeros metacrílicos como BisGMA, TEGDMA, monómeros metacrílicos fosfatados estabilizantes, canforquinona, co-iniciador, carga nanométrica de dióxido de silicio
FGM Productos Odontológicos (Joinville Brasil)

FGM Productos Odontoló gicos (Joinville Brasil)

$$
\begin{gathered}
\text { Bio-Art (São Carlos - Brasil) } \\
\text { KG Sorensen (São Paulo - Brasil) } \\
1014
\end{gathered}
$$

Monobond, Ivoclar Vivadent, Schaan, Liechtenstein

Dental Morelli (Sorocaba - Brasil)

Dental Morelli (Sorocaba - Brasil)

Orthocem, FGM Productos Odontológicos, (Joinville - Brasil)

Bluephase, Ivoclar Vivadent, Schaan, Liechtenstein 
Se utilizó la cerámica a base de disilicato de litio con alta translucidez (IPS e.max CAD / CAM - HT). Los 80 especímenes $(15 \mathrm{~mm}$ de ancho $\times 13 \mathrm{~mm}$ de altura $\times 2 \mathrm{~mm}$ de espesor) fueron producidos a partir de bloques cerámicos seccionados en rebanadas utilizando máquina de corte (CUTMASTER Erios, SP, BRASIL) con disco diamantado $(15,2 \times 0,5 \mathrm{~mm})$ (Buehler, New York, USA), en baja rotación (240 rpm), riego abundante y carga de $100 \mathrm{kgf}$. En el caso de que se produzca un cambio en la calidad de los alimentos, se debe tener en cuenta que el uso de la leche materna, segundos hasta los espesores deseados de $2,0 \mathrm{~mm}$.

A continuación, las muestras se llevaron al aparato de ultrasonido durante 3 minutos para eliminar los residuos. Después del pulido, las pastillas fueron cristalizadas en un horno cerámico (Programat EP3010, Ivoclar Vivadent) con una temperatura de 840$850{ }^{\circ} \mathrm{C} / 1544-1562{ }^{\circ} \mathrm{F}$.

En el centro de un dispositivo confeccionado en madera con base cuadrada de $62 \mathrm{~mm}^{2}$ para mayor estabilidad, y el centro circular con diámetro de $26 \mathrm{~mm}$ y altura, de $3 \mathrm{~mm}$ para adaptar el caño de PVC de forma estable (superficie de la cerámica en una posición paralela a la base del anillo del PVC). Un cilindro de PVC de $3 / 4$ de pulgada (Tigre de Brasil, Joinville, Brasil) de $1,5 \mathrm{~cm}$ de altura fue posicionado sobre el bloque cerámico que está encajado en el dispositivo de madera y fue incluida resina acrílica químicamente activada de color transparente Jet Set (Vibrator,
Vibramax Gold Line, Essence Dental Vh, Araraquara, SP, Brasil), proporcionada y preparada de acuerdo con la recomendación del fabricante.

Las 80 pastillas fueran divididas aleatoriamente en 8 grupos, según tratamiento de superficie y tipo de bracket, y subdivididas en termocicladas o no termocicladas, constituyendo 6 grupos experimentales, cada grupo con 5 muestras (Tabla II).

El tratamiento superficial de las pastillas cerámicas varía según los protocolos descritos a continuación:

Ácido fosfórico 37 \% (HF): Ácido fosfórico a $37 \%$ por 30 segundos, lavado por 30 segundos aplicación de silano y el secado durante 60 segundos (Ahrari et al.; Fornaini et al.; Mirhashemi et al.).

Ácido fluorhídrico $10 \%$ : Ácido fluorhídrico al $10 \%$ durante 30 segundos, lavado por 30 segundos, secado y aplicación de silano durante 60 segundos (Haydar et al., 1999; Gonçalves et al., 2011; Alakus \& Erturk, 2016).

Chorreado con óxido de aluminio (SB): chorreado con óxido de aluminio por 10 el segundo (Microjato Standard, Bio-Art, Jardim Tangará São Carlos, SP, Brasil) en $10 \mathrm{~mm}$ de distancia en presión de 3,5 a 4,5 (partículas de $50 \mathrm{~mm}$ ), seguido de lavado por 30 segundos, secado y aplicación de silano por 60 segundos (Della Bona et al.).

Tabla II. Grupos experimentales conforme las variables del estudio.

\begin{tabular}{|c|c|c|c|c|}
\hline $\begin{array}{l}\text { Superficie } \\
\text { cerámica }\end{array}$ & $\begin{array}{l}\text { Tratamiento de } \\
\text { superficie }\end{array}$ & $\begin{array}{c}\text { Bracket } \\
\text { ortodoncia }\end{array}$ & $\begin{array}{l}\text { Termociclación } \\
\text { (Si o No) }\end{array}$ & $\begin{array}{l}\text { Grupos Experimentales } \\
\qquad(\mathrm{n}=5)\end{array}$ \\
\hline \multirow{16}{*}{ e.max CAD } & \multirow{4}{*}{$\begin{array}{l}\text { Ácido fosfórico } 37 \% \\
\text { (HP) }\end{array}$} & \multirow{2}{*}{ Metálico } & $S$ & HPMS \\
\hline & & & $\mathrm{N}$ & HPMN \\
\hline & & \multirow{2}{*}{ Cerámico } & $S$ & HPCS \\
\hline & & & $\mathrm{N}$ & HPCN \\
\hline & \multirow{4}{*}{$\begin{array}{c}\text { Ácido fluorhídrico } \\
10 \%(\mathrm{HF})\end{array}$} & \multirow{2}{*}{ Metálico } & $S$ & HFMS \\
\hline & & & $\mathrm{N}$ & HFMN \\
\hline & & \multirow{2}{*}{ Cerámico } & $S$ & HFCS \\
\hline & & & $\mathrm{N}$ & HFCN \\
\hline & \multirow{4}{*}{$\begin{array}{l}\text { Chorreado con óxido } \\
\text { de aluminio (SB) }\end{array}$} & \multirow{2}{*}{ Metálico } & $S$ & SBMS \\
\hline & & & $\mathrm{N}$ & SBMN \\
\hline & & \multirow{2}{*}{ Cerámico } & $S$ & SBCS \\
\hline & & & $\mathrm{N}$ & SBCN \\
\hline & \multirow{4}{*}{$\begin{array}{l}\text { Asperación con punta } \\
\text { diamantada (DW) }\end{array}$} & \multirow{2}{*}{ Metálico } & $S$ & DWMS \\
\hline & & & $\mathrm{N}$ & DWMN \\
\hline & & \multirow{2}{*}{ Cerámico } & $S$ & DWCS \\
\hline & & & $\mathrm{N}$ & DWCN \\
\hline
\end{tabular}


PEIXOTO, N. M.; DE MATOS, J. D. M.; ANDRADE, V. C.; BOTTINO, M. A.; ZOGHEIB, L. V. Evaluación de la resistencia de unión de brackets ortodónticos fijados a cerámica de disilicato de lítio. Int. J. Odontostomat., 13(2):207-218, 2019.

Tabla III. Índice Remanente Adhesivo. Adaptado de Artun \& Bergland (1984).

\begin{tabular}{ll}
\hline & \multicolumn{1}{c}{ IRA (Índice de Remaneciente Adhesivo) } \\
\hline PUNTAJE & Frecuencia del Adhesivo Remanente \\
0 & Ausencia de cualquier residuo de la capa adhesiva en la superficie cerámica \\
1 & Presencia de menos de la mitad de la resina remanente en la superficie cerámica \\
2 & Presencia de más de la mitad de la resina remanente en la superficie cerámica \\
3 & $\begin{array}{l}\text { Presencia de toda la resina remanente en la superficie cerámica, junto con la impresión } \\
\text { del diseño de la base del brackets }\end{array}$ \\
\hline
\end{tabular}

Asperización con punta diamantada (DW): Asperación con punta diamantada 1014 con dos movimientos de ida y vuelta durante 2 segundos, de acuerdo con la marca en la base del bracket porcelana lavado por 30 segundos, secado y aplicación de silano por 60 segundos. Cada cinco pastillas se utilizó una nueva broca.

La aplicación de silano (Monobond, Ivoclar Vivadent) en los grupos se dio a través de una gota dispensada en un pote Dappen y, a continuación, se aplicó una fina capa sobre la superficie cerámica (Miyazaki et al., 2009).

En el presente estudio se utilizaron Brackets ortodónticos del tipo Edgwise (Morelli, Sorocaba / SP, Brasil), metálicos y cerámicos, para incisivos centrales superiores, de pequeñas dimensiones $\left(9 \mathrm{~mm}^{2}\right)$. Estos brackets presentan bases relativamente planas y retenciones con forma de ranura, permitiendo así una mejor adaptación a la superficie de la cerámica y un posicionamiento más estable. En la base de los brackets se aplicó resina ortodóntica (Orthocem, FGM, Joinville, Brasil) y estos fueron colocados en el centro de las plaquitas cerámicas de manera estandarizada por medio de la medición con regla milimetrada y pánx universal digital.

Los brackets fueron fijados en el centro del cuerpo de prueba con presión estandarizada utilizando carga de 500 gramos mantenidos por 10 segundos por medio de un dispositivo fabricado para este propósito. En la aplicación de la presión, el exceso de material fue removido con la ayuda de una espátula para resina y la polimerización fue activada durante 40 segundos (tiempo indicado por el fabricante) siendo 10 segundos en cada uno de los márgenes del soporte, con el aparato fotopolimerizador Bluephase, densidad de potencia por encima de $1100 \mathrm{~mW} / \mathrm{cm}^{2}$. La densidad de potencia de la fuente fotoactivadora se determinó por medio de un radiómetro. Se concluyó la cementación de los cuerpos de prueba en un contenedor de vidrio separado por grupo, sumergidos en $10 \mathrm{ml}$ de agua destilada y llevados a un invernade- ro a $37^{\circ} \mathrm{C}$ durante 24 horas antes de la termociclación y la prueba de cizallamiento.

Los cuerpos de prueba fueron sometidos a la termociclación, donde los mismos pasaron por 500 ciclos térmicos con variaciones de temperatura entre $5{ }^{\circ} \mathrm{C}$ a $55^{\circ} \mathrm{C}$ (ISO, 2015) (Caroline, 2015), y el tiempo de transferencia de 10 segundos entre los banos en agua desionizada (Trites et al., 2004; Yuasa et al., 2010; Jurubeba et al., 2017).

Las pruebas se realizaron en una máquina de ensayo universal (EMIC 2000, São José dos Pinhais / PR, Brasil) usando una célula de carga de $500 \mathrm{kgf}$ y una velocidad de $1 \mathrm{~mm}$ por minuto hasta el fallo. Los cuerpos de prueba fueron colocados en la matriz, quedando solamente el brackets expuesto a la fuerza aplicada por la punta activa en su parte superior generando una resistencia al cizallamiento en la interfaz hasta la ruptura, cuando los valores en kgf fueron anotados, convertidos en MPa utilizando la siguiente fórmula: $\mathrm{MPa}=\left(\mathrm{Kgf} \times 0,9 \mathrm{~m} / \mathrm{s}^{2}\right) /\left(\right.$ área del brackets $\left.\mathrm{mm}^{2}\right)$.

Después de la prueba de cizallamiento, las superficies de los brackets y de la cerámica fueron analizadas con lupa estereoscópica con aumento de ocho veces, buscando visualizar la cantidad de adhesivo residual en las superficies, determinando la localización y modo de fallo clasificado de acuerdo con el Índice de Remanente Adhesivo (IRA) (Adhesive remnant index ARI) descrito por Artun \& Bergland (1984), según los escores presentados en la Tabla III. Los datos obtenidos por el índice auxilian en la caracterización de las fallas adhesivas por medio de la evaluación de las interfaces, permitiendo la comparación entre los grupos.

Con el fin de caracterizar en mayor aumento el patrón topográfico de la superficie cerámica tratada por los diferentes tratamientos, HP (ácido fosfórico), HF (ácido hidrofluorhídrico), DW (desgaste con broca) y SB (chorreado con óxido de aluminio), una muestra de cada uno el tratamiento fue seleccionado, según diferentes patrones de fallo, y fueron sometidas a microscopía electrónica de barrido (MEV) (Fig. 2). 
Los datos fueron analizados a través del software IBM SPSS versión 23. La frecuencia absoluta y relativa y la media y desviación estándar se calcularon para las variables cualitativas y cuantitativas, respectivamente. Los roles de medidas cuantitativas fueron evaluados en cuanto a la normalidad por medio de la prueba de Shapiro-Wilk y se observó no normalidad $(p<0,001)$. Por lo tanto, debido a la no normalidad de los datos, las pruebas de Mann-Whitney y Kruskal-Wallis se emplearon para verificar la asociación entre las variables de tratamiento de superficie, tipo de bracket (metálico o cerámico) y termociclado. Se utilizó un nivel de significancia del $5 \%$ en todos los análisis estadísticos.

\section{RESULTADOS}

Prueba de resistencia al cizallamiento. Los valores medios de resistencia de unión en MPa de cada grupo se ilustran en la Figura 1.

La estadística descriptiva (médias y desviación estándar) de los dados de resistencia de unión por las variables del estudio están representados en la Tabla IV.

En cuanto al tratamiento de la cerámica, numéricamente el grupo HF fue superior $(17,27 \pm 10,07$ $\mathrm{MPa}$ ), pero sin diferencia estadística con los demás tratamientos de superficie. En cuanto al tipo de bracket, el metálico fue superior $(22,79 \pm 9,15 \mathrm{MPa})$ a la del bracket del tipo cerámico $(8,32 \pm 4,15 \mathrm{MPa})$, con diferencia estadística $(p<0,001)$. La variable termociclaje resultó en discreta disminución de la resistencia de unión, pero sin significancia estadística.

Análisis de fallas (Tabla V). El patrón de fallo característico predominante del grupo HP para bracket metálico según el IRA, fue el score 1, señalando alguna eficiencia del tratamiento de superficie, ya que se verificó presencia de menos de la mitad del área de cementación con cemento resinoso remanente sobre la superficie cerámica.

El patrón de fallo característico predominante del grupo HP para bracket cerámico según el IRA, presentó puntuación 0 y 1 , señalando alguna eficiencia del tratamiento de superficie, ya que se verificó ausencia de cualquier residuo de la capa adhesiva en la superficie cerámica, así como, presencia de menos de la mitad del área de cemento con cemento resinoso remanente sobre la superficie cerámica.

Tabla IV. Distribución de los valores medios de resistencia de unión según las variables del estudio

\begin{tabular}{lccc}
\hline Variables & $\mathrm{n}(\%)$ & $\begin{array}{c}\text { Media } \pm \text { desviación } \\
\text { estándar (Mpa) }\end{array}$ & Valor p \\
\hline Grupo & & & 0,7242 \\
\hline HP & $20(25,0)$ & $15,07 \pm 10,61$ & \\
HF & $20(25,0)$ & $17,27 \pm 10,07$ & \\
SB & $20(25,0)$ & $15,49 \pm 10,09$ & \\
DW & $20(25,0)$ & $14,39 \pm 10,35$ & \\
\hline Tipo de bracket & & & $<0,0011$ \\
Metálico & $40(50,0)$ & $22,79 \pm 9,15$ & \\
Cerâmica & $40(50,0)$ & $8,32 \pm 4,15$ & \\
\hline Termociclación & & & 0,2771 \\
Si & $40(50,0)$ & $13,81 \pm 8,48$ & \\
No & $40(50,0)$ & $17,30 \pm 11,42$ & \\
\hline
\end{tabular}

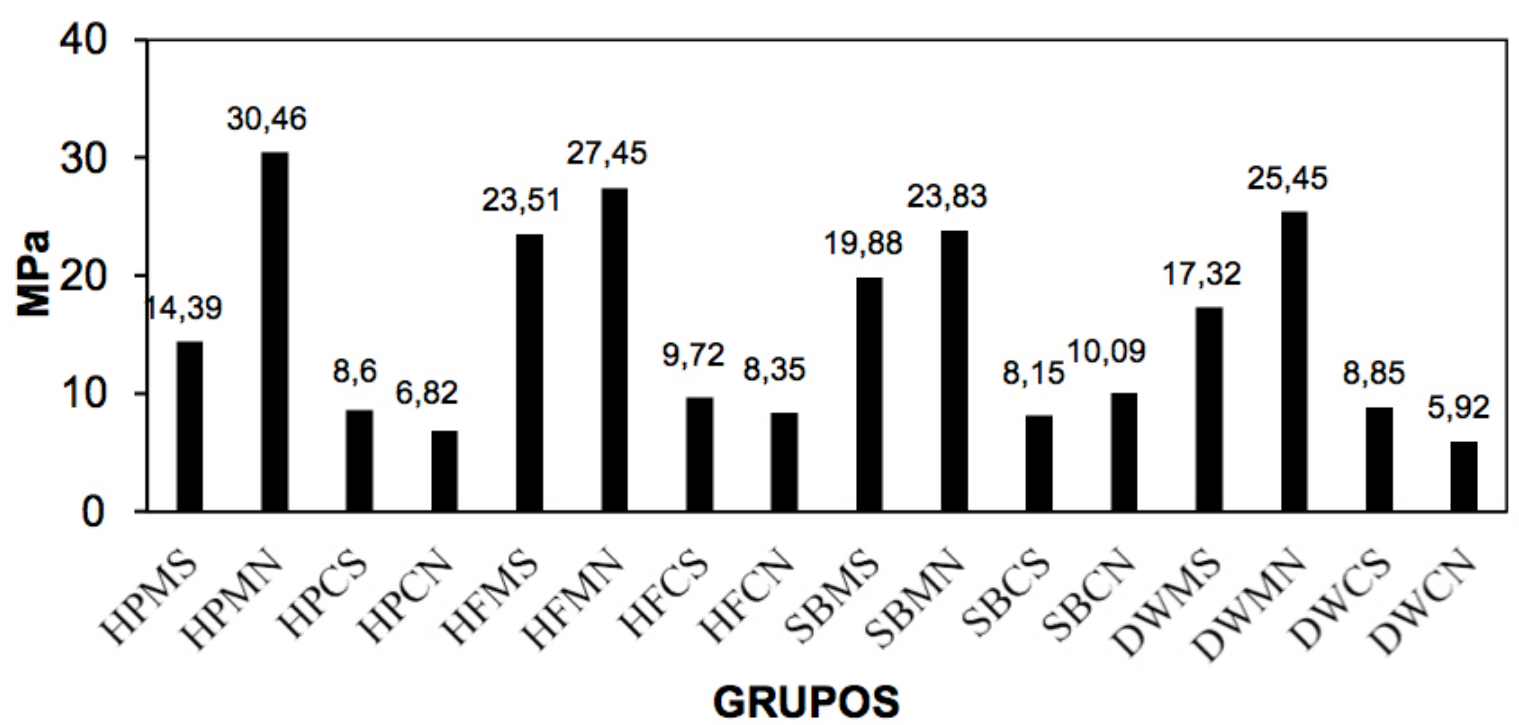

Fig. 1. Valores medios de resistencia de la unión por grupo. 
Tabla V. Distribución de fallas adhesivas por grupo según los escores.

\begin{tabular}{cccccc}
\hline & $\begin{array}{c}\text { Falla adhesiva } \\
\text { En la cerámica }\end{array}$ & $\begin{array}{c}\text { Falla } \\
\text { Mixto }\end{array}$ & $\begin{array}{c}\text { Falla } \\
\text { mixto }\end{array}$ & $\begin{array}{c}\text { Falla adhesive } \\
\text { en el brackets }\end{array}$ \\
\hline GRUPO & (IRA 0) & $($ IRA 1) & $($ IRA 2) & $($ IRA 3) & TOTAL \\
HPMS & 2 & 8 & - & - & 10 \\
HPMN & 6 & 4 & - & - & 10 \\
HPCS & 4 & 4 & 2 & - & 10 \\
HPCN & 2 & 2 & 2 & 4 & 10 \\
HFMS & - & 4 & - & 6 & 10 \\
HFMN & 2 & 8 & - & - & 10 \\
HFCS & - & 2 & 2 & 6 & 10 \\
HFCN & - & - & - & 10 & 10 \\
SBMS & 2 & 6 & 2 & - & 10 \\
SBMN & 2 & 6 & - & 2 & 10 \\
SBCS & - & 2 & - & 8 & 10 \\
SBCN & - & - & - & 10 & 10 \\
DWMS & - & - & 8 & 2 & 10 \\
DWMN & - & 6 & 4 & - & 10 \\
DWCS & - & - & 8 & 2 & 10 \\
DWCN & - & & - & 10 & 10
\end{tabular}
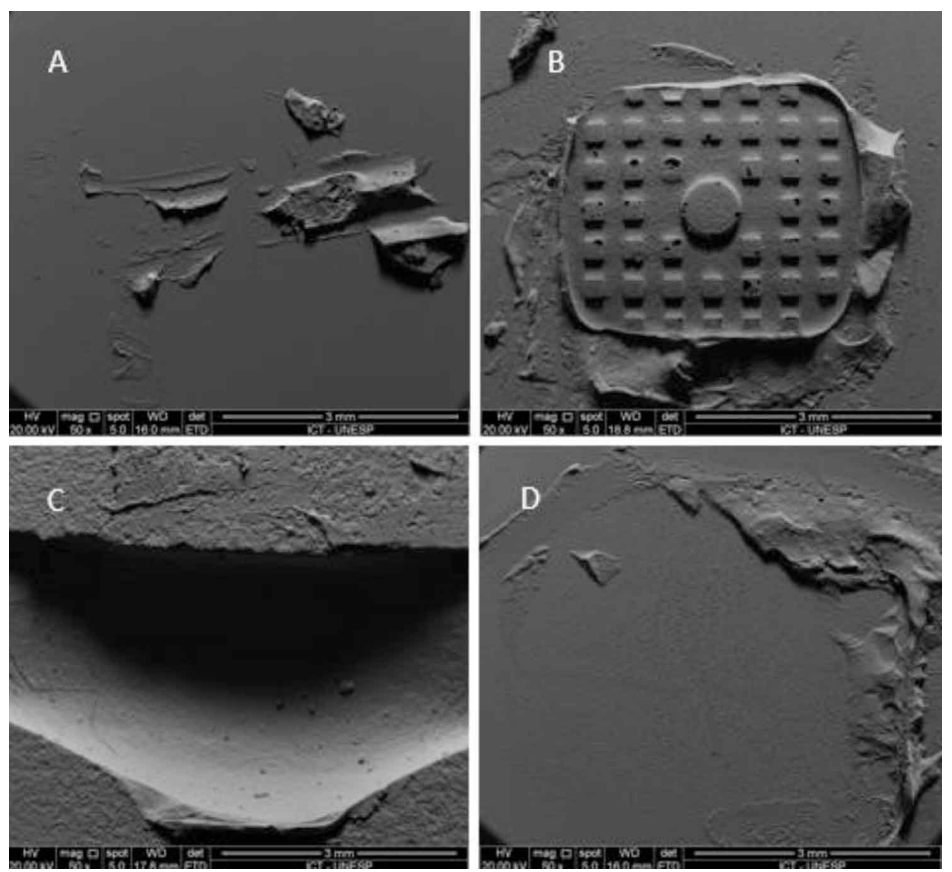

Fig. 2. Fotomicrografías de la superficie cerámica tras ensayo de resistencia de unión con brackets cerámicos y diferentes tratamientos de superficie. A. Ácido fosfórico al 37 \%; B. Ácido fluorhídrico al 10 $\%$; C. Cálculo con óxido de aluminio; D. Desgaste con broca.

El patrón de falla característico predominante de los brackets metálicos, en los grupos HF y SB, fue el score 1, verificando presencia de menos de la mitad de la resina remanente en la superficie cerámica. Mientras que el patrón de falla predominante de los brackets cerámicos en los grupos HF y SB fue el score 3, señalando mayor eficacia del tratamiento de superficie de la cerámica, en comparación con las demás puntuaciones 0,1 y 2, por la presencia de todo cemento resinoso remanente adherido a la superficie cerámica. Sin embargo, la fuerza de adhesión mayor puede comprometer el mantenimiento de la integridad de la porcelana.
El patrón de fallo característico predominante de los brackets metálicos en el grupo DW, fue el score 2, indicando presencia de más de la mitad de la resina remanente en la superficie cerámica. Mientras que el patrón de fallo de los brackets cerámicos en el grupo DW presentó puntuación 3 , señalado por la presencia de todo cemento resinoso remanente adherido a la superficie cerámica. Tal hecho evidenció la pobre unión adhesiva del cemento resinoso con la superficie de cementación del cemento bracket cerámico si se compara con la resistencia de unión con la superficie cerámica de disilicato de litio.

Microscopia electrónica de barrido (MEB). Diferentes patrones de fallo fueron encontrados en las muestras sometidas a la microscopía electrónica de barrido después de termociclado y ensayo de resistencia al cizallamiento, conforme presentado en la Figura 2.

\section{DISCUSIÓN}

La adhesión de brackets ortodónticos en la superficie cerámica es un procedimiento desafiante, considerando la resistencia suficiente frente a la fuerza ortodóntica y el mantenimiento de la integridad de la superficie de la restauración después de la remoción del brazalete. El tratamiento de la superficie cerámica se hace necesario para aumentar la fuerza de unión entre el bracket y la misma para resistir el movimiento ortodóntico durante la corrección de la mala oclusión. La fuerza de adhesión debe ser lo suficientemente alta para resistir el despegue accidental durante el tratamiento ortodóntico, y lo suficientemente baja para que la fuerza excesiva no necesite ser aplicada durante la remoción al final del tratamiento (Reynolds \& von Fraunhofer, 1976).

Esta investigación evaluó la resistencia al cizallamiento de brackets metálicos y cerámicos sobre una cerá- 
mica a base de disilicato teniendo como variables: tratamiento de la superficie cerámica, tipo de bracket y termociclaje. Se investigó entonces si el tratamiento de la superficie, a través de cuatro tratamientos distintos, el tipo de bracket, cerámico o metálico, y la realización o no de termociclado previo al procedimiento de pegado de los brackets influenciaría significativamente la resistencia de unión entre los brackets y la cerámica de disilicato de litio.

Considerando que la propuesta de este trabajo fue evaluar la efectividad de diferentes tratamientos de superficie de la cerámica, se utilizaron protocolos recomendados por diferentes estudios para cada tipo de tratamiento de superficie como el acondicionamiento con ácido fosfórico a $37 \%$ por 30 segundos (Ahrari et al.). El condicionamiento con ácido hidrofluorhídrico al 10 \% (Haydar et al.; Gonçalves et al.; Alakus et al., 2016) por 60 segundos; el chorreado con partículas de óxido de aluminio por 10 segundos (Della Bona et al.); y la asperación con broca por 2 segundos como control negativo.

La resistencia de unión considerada clínicamente aceptable para que el bracket resista el movimiento ortodóntico sin desprenderse está entre 6 a $8 \mathrm{MPa}$ (Reynolds \& von Fraunhofer; Gillis \& Redlich, 1998; Bourke et al., 1999). Considerando este parámetro, sólo los grupos HPCN y DWCN obtuvieron resistencia insuficiente (Fig. 1). Sin embargo, estos resultados deben interpretarse con cautela debido a las limitaciones inherentes a los ensayos de laboratorio que no necesariamente se confirman clínicamente (Zachrisson, 2000).

Otro parámetro de resistencia a considerar se refiere al tipo de fallo. Se ha señalado que, si la fuerza de adhesión entre la cerámica y la resina es superior a $13 \mathrm{MPa}$, la falla probablemente será cohesiva (Thurmond et al., 1994). Esta inferencia fue confirmada en este estudio para los brackets metálicos independientemente del termociclado y tratamiento de superficie (Fig. 1 y Tabla IV). El objetivo del condicionamiento ácido de cerámicas vítreas es promover irregularidades superficiales, a ser impregnadas con la aplicación de silano, y así aumentar la fuerza de unión entre el cemento y la cerámica (Barghi et al., 2000; Kumbuloglu et al., 2005; Taskonak \& Sertgöz, 2006; Fabianelli et al., 2010; Fasbinder et al., 2010).

El tratamiento de superficie con ácido fosfórico ha presentado ineficiencia para proporcionar retención mecánica en cerámica cuando se utilizan en combi- nación con resina compuesta (Cochran et al. 1997). En esta investigación, los grupos que utilizaron el ácido fosfórico como tratamiento de superficie, obtuvieron diferentes tipos de fallas con mayor predominio para las mixtas, considerando fallas en ambas interfaces bracket-cemento y cemento-cerámica después del despegue. Se llama atención para la mayor cantidad de fallas adhesivas en la cerámica cuando comparadas al mismo tipo de fallo visto en los demás tratamientos (Tabla III). Sin embargo, analizando conjuntamente la resistencia al cizallamiento promedio de sólo grupos con soporte metálico, que se obtiene una fuerza de unión clínicamente aceptable (Reynolds \& von Fraunhofer; Gillis \& Redlich; Bourke et al., 1999).

El ácido hidrofluorhídrico, con su efecto corrosivo sobre la matriz vítrea, es nocivo e irritante para los tejidos blandos, lo que hace que el producto de uso estricto por los ortodoncistas (Barbosa et al., 1995). Sin embargo, su eficiencia en la mejora de la adhesión entre bracket y cerámica ha sido ampliamente aceptada en comparación con el ácido fosfórico (Zachrisson \& Buyukyilmaz, 1993; Barbosa et al.). Tal comportamiento se encuentra debido a su mayor eficacia en la disolución del cristalino, facilitando la retención micromecánica. Sin embargo, la superioridad numérica de los valores de resistencia de unión en la mayoría de los grupos que utilizaron el ácido fluorhídrico no fue estadísticamente significativa. Sin embargo, solamente se observó apenas dos fallas adhesivas sobre la superficie cerámica.

El tratamiento de la cerámica con chorreado de óxido de aluminio viene siendo muy utilizado por los profesionales y objetiva también la promoción de irregularidades en la cerámica. Esta técnica apunta resultados satisfactorios que comprueban el aumento de la resistencia a la unión de los cementos a las cerámicas. En la mayoría de los casos, las variaciones en el tamaño de las partículas (generalmente entre 30 y $100 \mathrm{~mm}$ ), su aplicación, asociada o no al condicionamiento ácido y la posterior aplicación del silano (Borges et al., 2003; Spohr et al., 2003; Ozcan et al., 2008). Y en el caso de las mujeres, en el caso de las mujeres. En este estudio se utilizó el chorreado con partículas de $50 \mathrm{~mm}$ y silano. Los grupos que utilizaron granallado como tratamiento de superficie, presentaron valores numéricos similares y sin diferencia estadística si comparado a los demás tratamientos. A pesar de ello, se llama atención el bajo número de fallas adhesivas en la cerámica, señalando mayor alteración superficie y favorecimiento de la unión con el cemento resinoso. 
El tratamiento de la cerámica con desgaste de broca promueve la remoción del glaseado de la porcelana, así como los demás tratamientos, excepto el tratamiento con ácido fosfórico, creando así ranuras en su estructura, causando irregularidades y aumentando su retención entre la resina y la porcelana, las costas de algún daño estructural. Tales daños pueden ser contorneados con métodos de pulido de la porcelana (Kern \& Thompson, 1994), pero si el pulido de la superficie cerámica después de la finalización del tratamiento ortodóntico con la remoción del bracket no es suficiente para devolver la estética inicial de la restauración, el paciente tenderá a cambiarla (Smith et al., 1988). De esta forma, no debe ser una alternativa indicada en estas situaciones. De todas formas, este tratamiento de superficie fue utilizado en este estudio como control negativo. Considerando los promedios de los valores de resistencia de unión no hubo diferencia significativa con los demás grupos de tratamiento. Se llama atención a la ocurrencia de fallas adhesivas en la superficie cerámica (Tabla V).

Los estudios vienen mostrando que el área de la superficie adhesiva a ser probada está directamente relacionada al patrón de fallo obtenido. Así, área de superficies más amplias suelen resultar en mayor prevalencia de fallas cohesivas (Pashley et al., 1995). La sección tranversal de la muestra también parece influenciar la resistencia de unión. En estudio de Phrukkanon et al. (1998), la sección circular mostró resultar en una mayor resistencia adhesiva cuando se comparó la sección rectangular. Según la literatura, cuanto menor es la superficie del espécimen, menor es el área disponible para fallas, resultando en mejores resultados de resistencia de unión (Ghassemieh, 2008). Cuanto mayor sea el área de prueba, menor es la resistencia adhesiva, y que hasta $3 \mathrm{~mm}$ los resultados son más favorables para los ensayos de macrotración (Escribano et al., 2003). Para obtener el valor de la resistencia adhesiva, la carga se aplica en la interfaz adhesiva por medio de una punta, que está acoplada a una máquina de ensayo universal.

Así, por la división de la fuerza aplicada por el área adhesiva total se obtendrá la resistencia de unión inducida por la tensión de cizallamiento. Las pruebas de cizallamiento son las pruebas más frecuentemente empleadas para la evaluación de la resistencia adhesiva de materiales, esto probablemente se explique por la simplicidad del método y por la facilidad de adecuación a los diferentes equipos disponibles en los diversos laboratorios.
Los especímenes fueron sometidos a la termociclación con el fin de reproducir más de cerca el ambiente bucal simulando el envejecimiento de la muestra, generando estrés en la interfaz entre diferentes materiales con contracción y expansión diferentes cuando sometidos a calefacción y enfriamiento. Esto puede producir efectos negativos debido a los coeficientes disimilares de la expansión térmica entre los materiales. Se afirmó que un régimen de termociclado que contenía un mínimo de 500 ciclos en agua entre $5^{\circ} \mathrm{C}$ y $55^{\circ} \mathrm{C}$ sería una prueba de envejecimiento artificial suficiente (de Matos et al.). Debido a los diferentes hallazgos en la literatura, la necesidad de períodos más largos de termociclado sigue siendo una cuestión de discusión, no habiendo una estandarización en cuanto al número de ciclos que sería suficiente para reproducir el ambiente bucal. En nuestro estudio, no hubo una diferencia estadística entre los grupos termociclados y los no termociclados.

La durabilidad de la unión resina-cerámica se evalúa in vitro por termociclaje e inmersión en agua. La termociclación utiliza diferencias en los coeficientes térmicos de expansión del material cerámico y resinoso para estresar la unión adhesiva; mientras que el almacenamiento de agua evalúa la resistencia de la unión adhesiva a la degradación hidrolítica. Se realizaron varios estudios de termociclaje para evaluar la durabilidad de la unión cemento-cerámica. Además, los resultados de estos estudios no se pueden comparar debido al número de ciclos térmicos y al tipo de prueba de fuerza de adhesión.

Después del análisis estadístico de los resultados se verificó que, entre las variables estudiadas, sólo el tipo de bracket presentó diferencia estadística significativa (Tabla II). Las variables de tratamiento de superficie y termociclado, para los protocolos y el tamaño de las muestras utilizadas en esta investigación, no influenciaron significativamente en los valores de resistencia al cizallamiento entre brackets ortodónticos y la cerámica vítrea a base de disilicato de litio. Los mayores valores de resistencia de unión entre el bracket y la cerámica se observaron en los grupos donde se utilizó el brazalete metálico, siendo significativamente mayor que los grupos donde el brazalete cerámico fue utilizado, independiente del tratamiento de superficie y termociclaje.

El análisis cualitativo e IRA demuestra que los grupos HP y DW presentaron menor resistencia de unión adhesiva en la cerámica y mayor resistencia 
adhesiva en el brazalete, mientras que en los grupos HF y SB se observó mayor resistencia de unión en la cerámica y una menor falla adhesiva en el bracket.

Actualmente, jóvenes y adultos han buscado la ortodoncia para obtener una sonrisa armónica. Los pacientes fueron sometidos a otros tratamientos de rehabilitación previa, tales como el uso de prótesis dentales, por ejemplo, prótesis fija, removible o incluso facetas cerámicas. La ortodoncia no sólo implica el uso de aparatos para mejorar la apariencia general de los dientes, sino también para solucionar problemas funcionales causados por dientes desalineados. El protocolo y flujo de atención ortodóntico de primera elección recomiendan el inicio y la finalización del tratamiento en el período de la niñez y la adolescencia preferentemente, así como previamente a procedimientos restauradores definitivos. Sin embargo, por diferentes razones, tal secuencia cronológica de eventos, no siempre es posible de ejecutarse. Así, existe gran demanda de pacientes adultos con necesidad y deseo de alineamiento dental.

El foco de esta investigación se apoyó en una situación clínica atípica o no convencional, es decir, de la simulación de un tratamiento con brackets ortodónticos en pacientes ya portadores de facetas y / o coronas cerámicas. La dificultad de unión entre brackets cerámicos y estas superficies cerámicas, así como la limitación estética de los brackets convencionales por la cara vestibular, podrán ser contornadas con la ortodoncia lingual o los alineadores invisibles, pero no todos los pacientes con esta indicación podrán tener acceso a tales indicaciones alternativas de tratamiento justificando esta investigación de laboratorio.

Las hipótesis nulas que el tratamiento de superficie de la cerámica no causaría diferencia significativa de resistencia de unión entre los brackets y la cerámica a la base de disilicato de litio y que el termociclaje de los especímenes no causaría diferencia significativa de resistencia de unión entre los brackets y la cerámica la base de disilicato de litio fueron confirmadas, pero el tipo de brackets ortodóntico influenció significativamente la resistencia de unión con la superficie cerámica. Los nuevos estudios de laboratorio con nostalgia mayor y con tratamientos de la superficie de cementación del brackets cerámico pueden resultar en un mejor comportamiento de unión a las superficies de las restauraciones cerámicas.

\section{CONCLUSION}

Las variables de tratamiento de superficie y termociclado no influenciaron significativamente los valores de resistencia de unión. Sin embargo, el tipo de bracket, especialmente el cerámico, influenció negativamente los valores de resistencia de unión, así como los tipos de fallas, en el pegado de los mismos sobre superficies cerámicas de disilicato de litio. Así, queda evidente la necesidad de nuevas investigaciones, a fin de buscar alternativas de tratamiento superficial de los brackets cerámicos, que mejoren la previsibilidad de utilización de éstos sobre restauraciones cerámicas.

PEIXOTO, N. M.; DE MATOS, J. D. M.; ANDRADE, V. C.; BOTTINO, M. A. \& ZOGHEIB, L. V. Evaluation of union resistance of orthodontic bracelets fixed to lithium dissylate ceramic. Int. J. Odontostomat., 13(2):207-218, 2019.

ABSTRACT: To evaluate the shear strength of cemented orthodontic brackets to a dental ceramic based on lithium disilicate. Rectangular samples 80 based glassceramic lithium disilicate were prepared. The samples were initially divided into 4 groups of 20 discs according to surface treatment: $37 \%$ phosphoric acid for 30 seconds (HP); $10 \%$ hydrofluoric acid for 60 seconds (HF); blasting with aluminum oxide for 4 seconds (SB); and diamond-tipped for 10 seconds (DW). Each group was divided into two subgroups for the Edgwise bracket cementation: metallic and ceramic. The two new groups were subdivided into thermocyclers or nonthermocyclics. The thermocycled samples passed 500 cycles with temperature variations between $5^{\circ} \mathrm{C}$ to $55^{\circ} \mathrm{C}$. The numerical values obtained in the shear test were submitted to descriptive statistical analysis (mean and standard deviation), and the Shapiro-Wilk normality test and nonparametric Kruskal-Wallis and Mann-Whitney tests were performed. It was found that there was no statistically significant difference in the type of surface treatment and conducting or not thermocycling, observing statistically significant difference only on the bracket type, and the bond strength at higher metal bracket than in ceramics. Thus, the type of surface treatment and thermocycling aging were not decisive in the bond strength of orthodontic brackets to the lithium disilicate ceramic.

KEY WORDS: ceramic, orthodontic brackets, shear strength.

\section{REFERENCIAS BIBLIOGRÁFICAS}

Ahrari, F.; Heravi, F. \& Hosseini, M. CO2 laser conditioning of porcelain surfaces for bonding metal orthodontic brackets. Lasers Med. Sci., 28(4):1091-7, 2013 
Amoroso, A. P.; Ferreira, M. B.; Torcato, L. B.; Pellizzer, E. P.; Mazaro, J. V. Q. \& Gennari Filho, H. Cerâmicas odontológicas: propriedades, indicações e considerações clinicas. Rev. Odontol. Araçatuba, 33(2):19-25, 2012.

Artun, J. \& Bergland, S. Clinical trials with crystal growth conditioning as an alternative to acid-etch enamel pretreatment. Am. J. Orthod., 85(4):333-40, 1984.

Bakhadher, W.; Halawany, H.; Talic, N.; Abraham, N. \& Jacob, V. Factors affecting the shear bond strength of orthodontic brackets - A review of in vitro studies. Acta Medica (Hradec Kralove), 58(2):43- 8, 2015.

Barbosa, V. L.; Almeida, M. A.; Chevitarese, O. \& Keith, O. Direct bonding to porcelain. Am. J. Orthod. Dentofacial Orthop., 107(2):159-64, 1995.

Barghi, N.; Berry, T. \& Chung, K. Effects of timing and heat treatment of silanated porcelain on the bond strength. J. Oral Rehabil., 27(5):407-12, 2000.

Borges, G. A.; Sophr, A. M.; de Goes, M. F.; Sobrinho, L. C. \& Chan, D. C. Effect of etching and airborne particle abrasion on the microstructure of different dental ceramics. J. Prosthet. Dent., 89(5):479-88, 2003.

Brooks, S. A. \& Polk, M. Esthetic orthodontic considerations for the adult patient: a general dentist's perspective. J. Esthet. Dent., 10(6):305-8, 1998.

Cochran, D.; O'Keefe, K. L.; Turner, D. T. \& Powers, J. M. Bond strength of orthodontic composite cement to treated porcelain. Am. J. Orthod. Dentofacial Orthop., 111(3):297-300, 1997.

de Matos, N. R. S.; Costa, A. R.; Valdrighi, H. C.; Correr, A. B.; Vedovello, S. A.; Santamaria Jr., M. \& Correr-Sobrinho, L. Effect of acid etching, silane and thermal cycling on the bond strength of metallic brackets to ceramic. Braz. Dent. J., 27(6):734-8, 2016.

Della Bona, A. \& Di Guida, L. A. Evidências cientificas para a adesão de bráquetes cerâmicos a diferentes substratos odontológicos. Salusvita, 33(3):365-87, 2014.

Della Bona, A.; Anusavice, K. J. \& Shen, C. Microtensile strength of composite bonded to hot-pressed ceramics. J. Adhes. Dent., 2(4):305-13, 2000.

Ebert, T.; Elsner, L.; Hirschfelder, U. \& Hanke, S. Shear bond strength of brackets on restorative materials: Comparison on various dental restorative materials using the universal primer Monobond $\AA$ Plus. J. Orofac. Orthop., 77(2):73-84, 2016.

Escribano, N. I.; Del-Nero, M. O. \& de la Macorra, J. C. Inverse relationship between tensile bond strength and dimensions of bonded area. J. Biomed. Mater. Res. B Appl. Biomater., 66(1):419-24, 2003.

Fabianelli, A.; Pollington, S.; Papacchini, F.; Goracci, C.; Cantoro, A.; Ferrari, M. \& van Noort, R. The effect of different surface treatments on bond strength between leucite reinforced feldspathic ceramic and composite resin. J. Dent., 38(1):3943, 2010.

Fasbinder, D. J.; Dennison, J. B.; Heys, D. \& Neiva, G. A clinical evaluation of chairside lithium disilicate CAD/CAM crowns: a two-year report. J. Am. Dent. Assoc., 141 Suppl. 2:10S-4S, 2010.

Flores, D. A.; Caruso, J. M.; Scott, G. E. \& Jeiroudi, M. T. The fracture strength of ceramic brackets: a comparative study. Angle Orthod., 60(4):269-76, 1990.

Fornaini, C.; Merigo, E.; Vescovi, P.; Lagori, G. \& Rocca, J. Use of laser in orthodontics: applications and perspectives. Laser Ther., 22(2):115-24, 2013.

Gehrt, M.; Wolfart, S.; Rafai, N.; Reich, S. \& Edelhoff, D. Clinical results of lithium-disilicate crowns after up to 9 years of service. Clin. Oral Investig., 17(1):275-84, 2013.

Ghassemieh, E. Evaluation of sources of uncertainties in microtensile bond strength of dental adhesive system for different specimen geometries. Dent. Mater., 24(4):536-47, 2008.
Gillis, I. \& Redlich, M. The effect of different porcelain conditioning techniques on shear bond strength of stainless steel brackets. Am. J. Orthod. Dentofacial Orthop., 114(4):387-92, 1998.

Girish, P. V.; Dinesh, U.; Bhat, C. S. \& Shetty, P. C. Comparison of shear bond strength of metal brackets bonded to porcelain surface using different surface conditioning methods: an in vitro study. J. Contemp. Dent. Pract., 13(4):487-93, 2012.

Gonçalves, P. R.; Moraes, R. R.; Costa, A. R.; Correr, A. B.; Nouer, P. R.; Sinhoreti, M. A. \& Correr-Sobrinho, L. Effect of etching time and light source on the bond strength of metallic brackets to ceramic. Braz. Dent. J., 22(3):245-8, 2011.

Grewal Bach, G. K.; Torrealba, Y. \& Lagravère, M. O. Orthodontic bonding to porcelain: a systematic review. Angle Orthod., 84(3):555-60, 2014

Haydar, B.; Sarikaya, S. \& Cehreli, Z. C. Comparison of shear bond strength of three bonding agents with metal and ceramic brackets. Angle Orthod., 69(5):457-62, 1999.

International Organization for Standardization (ISO). ISO/TS 11405:2003 Dental materials -- Testing of adhesion to tooth structure. Geneva, International Organization for Standardization, ISO Central Secretariat, 2015. Disponible en: https://www.iso.org/standard/31486.html

Jurubeba, J. E. P.; Costa, A. R.; Correr-Sobrinho, L.; Tubel, C. A. M.; Correr, A. B.; Vedovello, S. A.; Crepaldi, M. V. \& Vedovello, M. F. Influence of thermal cycles number on bond strength of metallic brackets to ceramic. Braz. Dent. J., 28(2):206-9, 2017.

Kern, M. \& Thompson, V. P. Sandblasting and silica coating of a glass-infiltrated alumina ceramic: volume loss, morphology, and changes in the surface composition. J. Prosthet. Dent., 71(5):453-61, 1994.

Kumbuloglu, O.; Lassila, L. V.; User, A.; Toksavul, S. \& Vallittu, P. $\mathrm{K}$. Shear bond strength of composite resin cements to lithium disilicate ceramics. J. Oral Rehabil., 32(2):128-33, 2005.

Lestrade, A. M.; Ballard, R. W.; Xu, X.; Yu, Q.; Kee, E. L. \& Armbruster, P. C. Porcelain surface conditioning protocols and shear bond strength of orthodontic brackets. Aust. Orthod. J., 32(1):18-22, 2016.

Macri, R. T.; de Lima, F. A.; Bachmann, L.; Galo, R.; Romano, F. L.; Borsatto, M. C. \& Matsumoto, M. A. CO2 laser as auxiliary in the debonding of ceramic brackets. Lasers Med. Sci., 30(7):1835-41, 2015.

Masioli, D. L. C.; Almeida, M. D. O.; Masioli, M. A. \& Almeida, J. R. M. Assessment of the effect of different surface treatments on the bond strength of brackets bonded to acrylic resin. Dent. Press J. Orthod., 16(1):37-47, 2011.

Mehta, A. S.; Evans, C. A.; Viana, G.; Bedran-Russo, A. \& GalangBoquiren, M. T. S. Bonding of metal orthodontic attachments to sandblasted porcelain and zirconia surfaces. BioMed Res. Int., 2016:5762785, 2016.

Mirhashemi, A.; Sharifi, N.; Moharrami, M. \& Chiniforush, N. Evaluation of different types of lasers in surface conditioning of porcelains: a review article. J. Lasers Med. Sci., 8(3):10111, 2017.

Miyazaki, T.; Hotta, Y.; Kunii, J.; Kuriyama, S. \& Tamaki, Y. A review of dental CAD/CAM: current status and future perspectives from 20 years of experience. Dent. Mater. J., 28(1):44-56, 2009.

Oliveira, W. F.; Popoff, D. A. V. \& Júnior, A. R. S. Restaurações estéticas com dissilicato de lítio: relato de caso clínico. Buenos Aires, EFDeportes.com Revista Digital, 18(179), 2013. Disponible ens: https://www.efdeportes.com/efd179/ restauracoes-esteticas-com-dissilicato-de-litio.htm

Ozcan, M.; Nijhuis, H. \& Valandro, L. F. Effect of various surface conditioning methods on the adhesion of dual-cure resin cement with MDP functional monomer to zirconia after thermal aging. Dent. Mater. J., 27(1):99-104, 2008. 
Pashley, D. H.; Sano, H.; Ciucchi, B.; Yoshiyama, M. \& Carvalho, R. M. Adhesion testing of dentin bonding agents: a review. Dent. Mater., 11(2):117-25, 1995.

Phrukkanon, S.; Burrow, M. F. \& Tyas, M. J. The influence of crosssectional shape and surface area on the microtensile bond test. Dent. Mater., 14(3):212-21, 1998.

Reynolds, I. R. \& von Fraunhofer, J. A. Direct bonding of orthodontic attachments to teeth: the relation of adhesive bond strength to gauze mesh size. Br. J. Orthod., 3(2):91-5, 1976.

Smith, G. A.; Mclnnes-Ledoux, P.; Ledoux, W. R. \& Weinberg, R. Orthodontic bonding to porcelain--bond strength and refinishing. Am. J. Orthod. Dentofacial Orthop., 94(3):245-52, 1988.

Spohr, A. M.; Sobrinho, L. C.; Consani, S.; Sinhoreti, M. A. \& Knowles, J. C. Influence of surface conditions and silane agent on the bond of resin to IPS Empress 2 ceramic. Int. J. Prosthodont., 16(3):277-82, 2003

Taskonak, B. \& Sertgöz, A. Two-year clinical evaluation of lithiadisilicate-based all-ceramic crowns and fixed partial dentures. Dent. Mater., 22(11):1008-13, 2006

Thurmond, J. W.; Barkmeier, W. W. \& Wilwerding, T. M. Effect of porcelain surface treatments on bond strengths of composite resin bonded to porcelain. J. Prosthet. Dent., 72(4):355-9, 1994.

Trites, B.; Foley, T. F. \& Banting, D. Bond strength comparison of 2 self-etching primers over a 3-month storage period. Am. J. Orthod. Dentofacial Orthop., 126(6):709-16, 2004.

Tysowsky, G. W. The science behind lithium disilicate: a metal-free alternative. Dent. Today, 28(3):112-3, 2009.

Vieira, S.; Saga, A.; Wieler, W. \& Maruo, H. Adesão em ortodontia Parte 2. Colagem em superfícies de amálgama, ouro e porcelana. J. Bras. Ortodon. Ortop. Facial., 7(41):415-24, 2002.

Yuasa, T.; lijima, M.; Ito, S.; Muguruma, T.; Saito, T. \& Mizoguchi, I. Effects of long-term storage and thermocycling on bond strength of two self-etching primer adhesive systems. Eur. J. Orthod., 32(3):285-90, 2010.

Zachrisson, B. U. \& Buyukyilmaz, T. Recent advances in bonding to gold, amalgam and porcelain. J. Clin. Orthod., 27(12):661-75, 1993.

Zachrisson, B. U. Orthodontic bonding to artificial tooth surfaces: clinical versus laboratory findings. Am. J. Orthod. Dentofacial Orthop., 117(5):592-4, 2000.

Zhang, Z. C.; Giordano, R.; Shen, G.; Chou, L. L. \& Qian, Y. F. Shear bond strength of an experimental composite bracket. J. Orofac. Orthop., 74(4):319-31, 2013.

Zhang, Z. C.; Qian, Y. F.; Yang, Y. M.; Feng, Q. P. \& Shen, G. Bond strength of metal brackets bonded to a silica-based ceramic with light-cured adhesive : Influence of various surface treatment methods. J. Orofac. Orthop., 77(5):366-72, 2016.
Dlrección para correspondencia:

Lucas Villaça Zogheib D.D.S.; M.D.; Ph.D.

Profesor de Prótesis Dentales

Departamento de Odontología

Universidade de Fortaleza UNIFOR

Avenida Washington Soares, 1321

Edson Queiroz

Fortaleza - CE

BRAZIL

Email: matosjefferson19@gmail.com

Recibido: 01-12-2018

Aceptado: 08-02-2019 